ANNALES

POLONICI MATHEMATICI

$94.3(2008)$

\title{
Meromorphic solutions of a kind of functional equations of Diophantine type
}

\author{
by Ping Li and Xianyang Zhang (Hefei)
}

\begin{abstract}
We apply Nevanlinna's value distribution theory to show that some functional equations of Diophantine type have no admissible meromorphic solutions. This result confirms a recent conjecture of $\mathrm{Li}$ and Yang.
\end{abstract}

1. Introduction and result. In the $1920 \mathrm{~s}, \mathrm{R}$. Nevanlinna developed the so-called value distribution theory which enables one to give an elementary proof of Picard's theorem and Borel's theorem. Nevanlinna's theory mainly consists of the so-called first and second fundamental theorems, expressed in terms of three quantities $T(r, f), m(r, f)$ and $N(r, f)$ associated with a given meromorphic function $f$; they are called the characteristic function, the proximate function and the counting function of $f$, respectively. A meromorphic function $a(z)$ is called a small function of $f$ if $T(r, a)=o(T(r, f))$, as $r \rightarrow \infty$, outside possibly an exceptional set of $r$ values of finite linear measure. The study of the value distribution theory has been extended to algebroid functions, meromorphic mappings, complex geometry and differential geometry. Also the value distribution theory is of great importance in the research of nonlinear differential equations. We know that the two fundamental theorems remain valid when the values $a_{i}$ are replaced by $a_{i}(z)$, which are small functions of $f$. By applying Nevanlinna's second fundamental theorem, it is easy to prove that Fermat's type functional equation

$$
a_{1} f^{n}+a_{2} g^{n}=1
$$

has no pair $(f, g)$ of admissible meromorphic solutions when $n \geq 4$, where $a_{1}$ and $a_{2}$ are two given meromorphic functions which do not vanish identically. By "admissible" we mean that all coefficients are small functions of the solutions. If $n=3$, then the above equation may have some elliptic functions

2000 Mathematics Subject Classification: 30D35, 30D05.

Key words and phrases: meromorphic function, functional equation, Nevanlinna theory. 
as its solutions. If $n=2$, then the above equation may have entire solutions, for example, $\sin ^{2} z+\cos ^{2} z=1$.

Recently, P. Li and C.-C. Yang considered the following Diophantine type equation:

$$
f^{n}+a_{1} f^{m}+a_{2}=a_{3} g^{k},
$$

and proved the following result.

Theorem A ([4], [5]). Suppose that $a_{1}, a_{2}, a_{3}$ are meromorphic functions not vanishing identically. If the positive integer triple $(n, m, k)$ satisfies $k>1, m<n$, and $n>k(m+2) /(k-1)$ or $n<k(m-2)$, then equation (1) has no pair of admissible meromorphic solutions. In addition, the equation has no pair $(f, g)$ of admissible meromorphic solutions when $n=5, m=2$, $k=4$.

By Theorems 2 and 3 in [6], equation (1) may have admissible solutions $(f, g)$ when $n \leq 4$ and $k \leq 4$. Moreover, we can show easily that (1) has no pair of admissible solutions when $n=5, m=2$ or 3 , and $k \geq 3$, in the case where $a_{1}, a_{2}, a_{3}$ are constants. It is then conjectured in [4] that the conclusion of Theorem A remains true when $k \geq 3, n=5, m=2$ or 3 . The purpose of the present paper is to confirm this conjecture by proving the following result.

THEOREM 1. Suppose that $a_{1}, a_{2}, a_{3}$ are meromorphic functions not vanishing identically, and $k, m(k \geq 3, m \leq 4)$ two positive integers. Then the functional equation

$$
f^{5}+a_{1} f^{m}+a_{2}=a_{3} g^{k}
$$

has no pair $(f, g)$ of admissible meromorphic solutions.

REMARK. Equation (2) may have meromorphic solutions when $k=2$. For example, let $f$ be a solution of the Weierstrass elliptic equation $\left(f^{\prime}\right)^{2}=$ $f^{3}+6 f^{2}+12 f+18$. One can show easily that $f^{5}-15 f^{3}+162=\left((f-3) f^{\prime}\right)^{2}$.

From Theorem A we see that equation (1) has no pair of admissible meromorphic solutions when $n=4, k=5, m=1$ or 3 . However, Theorem A cannot tell us whether (1) has admissible meromorphic solutions when $(n, m, k)=(4,2,5)$. By the method similar to that in the proof of Theorem 1 , we can prove that (1) has no pair of admissible meromorphic solutions when $(n, m, k)$ is any of the following triples: $(7,2,2),(7,5,2),(9,3,2),(9,6,2)$, $(10,3,2),(10,7,2),(6,2,3),(6,4,3),(8,4,3),(9,4,3),(9,5,3)$ and $(4,2,5)$. Moreover, we can prove that (1) has no pair of admissible meromorphic solutions when $n=2(m+2)$ and $k=2$ for $m \geq 5$.

By Nevanlinna's second fundamental theorem, one can show easily that equation (1) has no admissible meromorphic solutions when $a_{1}, a_{2}$ are constants and $(n, m, k)$ is one of the following triples: $(9,4,2),(9,5,2),(10,4,2)$, 
$(10,6,2),(7,3,2),(7,4,2),(8,3,2)$ and $(8,5,2)$. However, we do not know whether (1) has admissible meromorphic solutions when $(n, m, k)$ is one of these triples and $a_{1}, a_{2}$ are not constants.

2. Some lemmas. We need the following lemmas to prove the theorem. The first one is a slight improvement of a result in [7]. The second one is an improvement of Clunie's lemma. The third one is a generalization of the Malmquist-Yosida theorem.

LeMma 1. Let $f$ be a transcendental meromorphic function in the complex plane. Suppose that $P_{n}(f)$ is a polynomial in $f$ of degree $n$, and $Q_{1}(f)$, $Q_{2}(f)$ are differential polynomials in $f$ not identically zero. Let

$$
F:=P_{n}(f) Q_{1}(f)+Q_{2}(f) .
$$

Then

$$
\begin{aligned}
\left(n-\gamma_{Q_{2}}\right) T(r, f) \leq & \bar{N}(r, 1 / F)+\bar{N}_{0}\left(r, 1 / P_{n}(f)\right) \\
& +\left(\Gamma_{Q_{2}}-\gamma_{Q_{2}}+1\right) \bar{N}(r, f)+S(r, f),
\end{aligned}
$$

where $\Gamma_{Q_{2}}$ and $\gamma_{Q_{2}}$ denotes the weight and degree of $Q_{2}$ respectively, and $\bar{N}_{0}\left(r, 1 / P_{n}(f)\right)$ denotes the reduced counting function of zeros of $P_{n}(f)$ that are not zeros of $F$.

Lemma 2 ([1], [2]). Let $P_{n}(f)$ be a polynomial in $f$ of degree $n$, and $Q_{1}(f)$ and $Q_{2}(f)$ be differential polynomials in $f$ with coefficients being small proximate functions of $f$, with $\operatorname{deg} Q_{2}(f) \leq n$. If $P_{n}(f) Q_{1}(f)=Q_{2}(f)$, then $m\left(r, Q_{1}(f)\right)=S(r, f)$.

REMARK. The conclusion of Lemma 2 remains true when $P_{n}(f)$ is a polynomial in $f$ of degree $n$ with the leading coefficient being a small function of $f$ and the other coefficients being small proximate functions of $f$.

Lemma 3 ([3, p. 258]). Suppose that $D(f)$ is a differential polynomial in $f$, and $P(f), Q(f)$ are polynomials in $f$ with all coefficients being small functions of $f$. If $D(f)=Q(f) / P(f)$, then $Q(f) / P(f)$ reduces to a polynomial in $f$.

LEMMA 4. Suppose that $f$ is a nonconstant meromorphic function, and $P(f), Q(f)$ are polynomials in $f$ with coefficients being small functions of $f$. Let a be a small function of $f$. If

$$
\frac{P\left(f^{2}\right)+f Q\left(f^{2}\right)}{f^{2}-a}
$$

reduces to a polynomial in $f$, then $P(a)=Q(a)=0$.

Proof. We rewrite the numerator of (3) as

$$
P\left(f^{2}\right)-P(a)+f\left(Q\left(f^{2}\right)-Q(a)\right)+P(a)+Q(a) f .
$$


Since both $P\left(f^{2}\right)-P(a)$ and $Q\left(f^{2}\right)-Q(a)$ have the factor $f^{2}-a$, we deduce that $f^{2}-a$ is a factor of $P(a)+Q(a) f$ under the given assumption. It follows that $P(a)=Q(a)=0$.

3. Proof of Theorem 1. The case $k \geq 6, m \leq 4$ and the case $k \geq 3$, $m=1$ or 4 are covered by Theorem A. When $k=5$, by dividing $f^{5}+a_{1} f^{m}+$ $a_{2} g^{k}=a_{3}$ by $f^{5}$, we see that the cases $m=2$ and $m=3$ are equivalent. Therefore, we only need to consider the following cases.

CASE 1: $m \leq 4, k=4$. We are going to prove that the functional equation

$$
f^{5}+a_{1} f^{m}+a_{2}=a_{3} g^{4}
$$

has no pair $(f, g)$ of admissible meromorphic solutions, where $a_{1}, a_{2}, a_{3}$ are small functions of $f$ and $a_{1} a_{2} \not \equiv 0$. Let $F=f^{5}+a_{1} f^{m}+a_{2}$. From Theorem A, we only have to consider the cases of $m=2$ and $m=3$. By Lemma 1, in both cases we get

$$
3 T(r, f) \leq \bar{N}(r, 1 / F)+\bar{N}(r, 1 / f)+\bar{N}(r, f)+S(r, f) .
$$

From equation (4), it is easily seen that $5 T(r, f)=4 T(r, g)+S(r, f)$, and all poles of $f$ that are not poles or zeros of $a_{1}, a_{2}$ and $a_{3}$ have multiplicities at least 4 . Therefore $\bar{N}(r, f) \leq \frac{1}{4} N(r, f)+S(r, f)$. It follows from (5) that

$$
\begin{aligned}
3 T(r, f) & \leq \bar{N}(r, 1 / g)+\bar{N}(r, 1 / f)+\frac{1}{4} N(r, f)+S(r, f) \\
& \leq T(r, g)+T(r, f)+\frac{1}{4} T(r, f)+S(r, f) \\
& \leq\left(\frac{5}{4}+1+\frac{1}{4}\right) T(r, f)+S(r, f) \\
& \leq \frac{5}{2} T(r, f)+S(r, f) .
\end{aligned}
$$

This is impossible.

CASE 2: $m=2, k=3$. We are going to prove that the equation

$$
f^{5}+a_{1} f^{2}+a_{2}=a_{3} g^{3}
$$

has no pair $(f, g)$ of admissible meromorphic solutions, where $a_{1}, a_{2}, a_{3}$ are small functions of $f$ and $a_{1} a_{2} \not \equiv 0$. Let $F=f^{5}+a_{1} f^{2}+a_{2}$. By Lemma 1 , we have

$$
3 T(r, f) \leq \bar{N}(r, 1 / F)+\bar{N}(r, 1 / f)+\bar{N}(r, f)+S(r, f) .
$$

From (6), we see that $5 T(r, f)=3 T(r, g)+S(r, f)$, and all poles of $f$ that are not poles or zeros of the coefficients have multiplicities at least 3 . Therefore 
$\bar{N}(r, f) \leq \frac{1}{3} N(r, f)+S(r, f)$. It follows that

$$
\begin{aligned}
3 T(r, f) & \leq \bar{N}(r, 1 / g)+\bar{N}(r, 1 / f)+\frac{1}{3} N(r, f)+S(r, f) \\
& \leq T(r, g)+T(r, f)+\frac{1}{3} T(r, f)+S(r, f) \\
& \leq\left(\frac{5}{3}+1+\frac{1}{3}\right) T(r, f)+S(r, f) \\
& \leq 3 T(r, f)+S(r, f) .
\end{aligned}
$$

Hence

$$
\begin{aligned}
T(r, f) & =N(r, f)+S(r, f), \\
T(r, 1 / f) & =\bar{N}(r, 1 / f)+S(r, f), \\
T(r, 1 / g) & =\bar{N}(r, 1 / g)+S(r, f) .
\end{aligned}
$$

From (7)-(9), we obtain

$$
\begin{aligned}
& m(r, f)=S(r, f), \quad m(r, 1 / g)=S(r, f), \quad m(r, 1 / f)=S(r, f), \\
& N(r, 1 / f)=\bar{N}(r, 1 / f)+S(r, f), \quad N(r, 1 / g)=\bar{N}(r, 1 / g)+S(r, f) .
\end{aligned}
$$

Since

$$
F^{\prime}=\frac{F^{\prime}}{F}\left(f^{5}+a_{1} f^{2}+a_{2}\right)=5 f^{4} f^{\prime}+a_{1}^{\prime} f^{2}+2 a_{1} f f^{\prime}+a_{2}^{\prime},
$$

we have

$$
f^{4} a=\frac{F^{\prime}}{F} a_{1} f^{2}+\frac{F^{\prime}}{F} a_{2}-a_{1}^{\prime} f^{2}-2 a_{1} f f^{\prime}-a_{2}^{\prime},
$$

where $a=5 f^{\prime}-\left(F^{\prime} / F\right) f$. It is easily seen that $a \not \equiv 0$, since otherwise we have $5 f^{\prime} / f=F^{\prime} / F$, which leads to $F=d f^{5}$, where $d$ is a nonzero constant; then $f^{5}+a_{1} f^{2}+a_{2}=d f^{5}$, which is impossible. From (6) and the definition of $a$, we see that poles of $a g$ come from poles of $f$, poles of $g$, or zeros of $F$. Because poles of $g$ come from poles of $f$ or of the coefficients, we only need to consider poles of $f$ and the zeros of $F$ which are not poles or zeros of $a_{1}$, $a_{2}$ and $a_{3}$. From (6), we see that the multiplicities of all poles of $f$ which are not poles or zeros of the coefficients are divisible by 3 . From (10), we see that any pole of $f$ of multiplicity $3 k$ is a zero of $a$ of multiplicity $6 k-1$. Such poles of $f$ must be poles of $g$ with multiplicity $5 k$. Thus poles of $f$ are not poles of $a g$. On the other hand, if a zero of $F$ is a pole of $a$, then it must be a simple pole. From the equation $F=a_{3} g$, we see that zeros of $F$ must be zeros of $g$ or of $a_{3}$. Therefore, zeros of $F$ cannot be poles of $a g$. Hence we have $N(r, a g)=S(r, f)$. Note that $m(r, f)=S(r, f)$ and $m(r, g)=S(r, f)$. By Lemma 2, we get $m(r, a)=S(r, f)$. It follows that $T(r, a g)=S(r, f)$, 
which means that $a g$ is a small function of $f$. Define $G=a F$. Then

$$
\begin{aligned}
G & =5 f^{\prime} F-F^{\prime} f \\
& =5 f^{\prime}\left(f^{5}+a_{1} f^{2}+a_{2}\right)-\left(5 f^{4} f^{\prime}+a_{1}^{\prime} f^{2}+2 a_{1} f f^{\prime}+a_{2}^{\prime}\right) f \\
& =\left(3 a_{1} f^{2}+5 a_{2}\right) f^{\prime}-\left(a_{1}^{\prime} f^{3}+a_{2}^{\prime} f\right),
\end{aligned}
$$

and thus $G^{3}=a^{3} F^{3}=a^{3} a_{3} g^{3} F^{2}=F^{2} h$, where $h=a_{3}(a g)^{3}$ is a small function of $f$. It follows that

$$
F^{2} h=\left(\left(3 a_{1} f^{2}+5 a_{2}\right) f^{\prime}-\left(a_{1}^{\prime} f^{3}+a_{2}^{\prime} f\right)\right)^{3} .
$$

Therefore,

$$
\frac{F^{2} h+\left(a_{1}^{\prime} f^{3}+a_{2}^{\prime} f\right)^{3}}{3 a_{1} f^{2}+5 a_{2}}=: D(f)
$$

which is a differential polynomial in $f$. By Lemma 3 we see that $D(f)$ reduces to a polynomial in $f$. The left-hand side of the above equation can be expressed as

$$
\frac{P\left(f^{2}\right)+f Q\left(f^{2}\right)}{3 a_{1}\left(f^{2}+5 a_{2} / 3 a_{1}\right)}
$$

where

$$
\begin{aligned}
& P(z)=h z^{5}+h\left(a_{1} z+a_{2}\right)^{2}, \\
& Q(z)=2 h z^{2}\left(a_{1} z+a_{2}\right)+z\left(a_{1}^{\prime} z+a_{2}^{\prime}\right)^{3} .
\end{aligned}
$$

By Lemma 4 , we have $P\left(-5 a_{2} / 3 a_{1}\right)=Q\left(-5 a_{2} / 3 a_{1}\right)=0$. Note that $h$ is not identically zero. It follows from $P\left(-5 a_{2} / 3 a_{1}\right)=0$ that $\left(a_{2} / 3\right)^{3}=4\left(a_{1} / 5\right)^{5}$. Substituting this into $Q\left(-5 a_{2} / 3 a_{1}\right)=0$ yields $a_{2}=0$, a contradiction. Hence equation (6) has no nonconstant meromorphic solutions.

Case 3: $m=3, k=3$. We are going to prove that the functional equation

$$
f^{5}+a_{1} f^{3}+a_{2}=a_{3} g^{3}
$$

has no pair $(f, g)$ of admissible meromorphic solutions, where $a_{1}, a_{2}, a_{3}$ are small functions of $f$ and $a_{1} a_{2} \not \equiv 0$. Set $F=f^{5}+a_{1} f^{3}+a_{2}$. By Lemma 1 , we still have

$$
3 T(r, f) \leq \bar{N}(r, 1 / F)+\bar{N}(r, 1 / f)+\bar{N}(r, f)+S(r, f) .
$$

By using an argument similar to Case 1, we see that (7)-(9) are still valid in this case. Therefore, we have

$$
\begin{aligned}
& m(r, f)=S(r, f), \quad m(r, 1 / g)=S(r, f), \quad m(r, 1 / f)=S(r, f), \\
& N(r, 1 / f)=\bar{N}(r, 1 / f)+S(r, f), \quad N(r, 1 / g)=\bar{N}(r, 1 / g)+S(r, f) .
\end{aligned}
$$


Taking the derivative of $F$, we get

$$
F^{\prime}=\frac{F^{\prime}}{F} F=5 f^{4} f^{\prime}+3 a_{1} f^{2} f^{\prime}+a_{1}^{\prime} f^{3}+a_{2}^{\prime}=f^{5} \frac{F^{\prime}}{F}+a_{1} f^{3} \frac{F^{\prime}}{F}+a_{2} \frac{F^{\prime}}{F} .
$$

It follows that

$$
f^{2} a=a_{2} \frac{F^{\prime}}{F}-a_{2}^{\prime},
$$

where $a=5 f^{2} f^{\prime}+3 a_{1} f^{\prime}+a_{1}^{\prime} f-f^{3} F^{\prime} / F-a_{1} f F^{\prime} / F$. It is easily seen that $a \not \equiv 0$, since otherwise we have $F^{\prime} / F-a_{2}^{\prime} / a_{2}=0$, and integrating this equation yields $F=d a_{2}$, where $d$ is a nonzero constant; then $f^{5}+a_{1} f^{3}+$ $a_{2}=d a_{2}$, which is impossible. By an argument similar to Case 1 , we have $T(r, a g)=S(r, f)$. Hence $a g$ is a small function of $f$. Set $G=a F$. We have

$$
\begin{aligned}
G & =5 f^{2} f^{\prime} F+3 a_{1} f^{\prime} F+a_{1}^{\prime} f F-f^{3} F^{\prime}-a_{1} f F^{\prime} \\
& =\left(5 f^{2} a_{2}+3 a_{1} a_{2}\right) f^{\prime}-\left(a_{2}^{\prime} f^{3}+a_{1} a_{2}^{\prime} f-a_{1}^{\prime} a_{2} f\right) .
\end{aligned}
$$

From (13), we get $G^{3}=a^{3} F^{3}=a_{3}(a g)^{3} F^{2}=h F^{2}$, where $h=a_{3}(a g)^{3}$ is a small function of $f$. It follows that

$$
h F^{2}=\left(\left(5 f^{2} a_{2}+3 a_{1} a_{2}\right) f^{\prime}-\left(a_{2}^{\prime} f^{3}+a_{1} a_{2}^{\prime} f-a_{1}^{\prime} a_{2} f\right)\right)^{3} .
$$

Therefore,

$$
\frac{h F^{2}+\left(a_{2}^{\prime} f^{3}+a_{1} a_{2}^{\prime} f-a_{1}^{\prime} a_{2} f\right)^{3}}{5 a_{2} f^{2}+3 a_{1} a_{2}}=: D(f),
$$

which is a differential polynomial in $f$. By Lemma $3, D(f)$ reduces to a polynomial in $f$. We can rewrite the numerator of the left-hand side of (14) as $h P\left(f^{2}\right)+f Q\left(f^{2}\right)$, where

$$
\begin{aligned}
& P(z)=z\left(z^{2}+a_{2} z\right)^{2}+a_{2}^{2}, \\
& Q(z)=2 a_{2} h\left(z^{2}+a_{1} z\right)-z\left(a_{2}^{\prime} z+a_{1} a_{2}^{\prime}-a_{1}^{\prime} a_{2}\right)^{3} .
\end{aligned}
$$

By Lemma 4 , we have $P\left(-3 a_{1} / 5\right)=Q\left(-3 a_{1} / 5\right)=0$. That is,

$$
3\left(a_{1} / 5\right)^{5}=\left(a_{2} / 6\right)^{2}, \quad 50 h a_{1} a_{2}=3\left(2 a_{1} a_{2}^{\prime}-5 a_{1}^{\prime} a_{2}\right)^{3} .
$$

It follows from the above equations that $h a_{1} a_{2}=0$. This is impossible. Hence equation (13) has no nonconstant meromorphic solution.

CASE $4: m=2, k=5$. We are going to prove that the functional equation

$$
f^{5}+a_{1} f^{2}+a_{2}=a_{3} g^{5}
$$

has no pair $(f, g)$ of admissible meromorphic solutions, where $a_{1}, a_{2}, a_{3}$ are small functions of $f$ and $a_{1} a_{2} \not \equiv 0$. Let $F=f^{5}+a_{1} f^{2}+a_{2}$. By Lemma 1 ,

$$
3 T(r, f) \leq \bar{N}(r, 1 / F)+\bar{N}(r, 1 / f)+\bar{N}(r, f)+S(r, f) .
$$


From (15), it is easily seen that $T(r, f)=T(r, g)+S(r, f)$. Therefore,

$$
\begin{aligned}
3 T(r, f) & \leq \bar{N}(r, 1 / g)+\bar{N}(r, 1 / f)+\bar{N}(r, f)+S(r, f) \\
& \leq T(r, g)+2 T(r, f)+S(r, f) \leq 3 T(r, f)+S(r, f) .
\end{aligned}
$$

Hence

$$
\begin{aligned}
T(r, 1 / f) & =\bar{N}(r, 1 / f)+S(r, f), \\
T(r, f) & =N(r, f)+S(r, f), \\
T(r, 1 / g) & =\bar{N}(r, 1 / g)+S(r, f) .
\end{aligned}
$$

From (16)-(18), we obtain

$$
\begin{aligned}
& m(r, f)=S(r, f), \quad m(r, 1 / g)=S(r, f), \quad m(r, 1 / f)=S(r, f), \\
& N(r, 1 / f)=\bar{N}(r, 1 / f)+S(r, f), \quad N(r, 1 / g)=\bar{N}(r, 1 / g)+S(r, f) .
\end{aligned}
$$

Since

$$
F^{\prime}=\frac{F^{\prime}}{F} F=5 f^{4} f^{\prime}+2 a_{1} f f^{\prime}+a_{1}^{\prime} f^{2}+a_{2}^{\prime}=\frac{F^{\prime}}{F}\left(f^{5}+a_{1} f^{2}+a_{2}\right),
$$

we have

$$
f^{4} a=\frac{F^{\prime}}{F}\left(a_{1} f^{2}+a_{2}\right)-2 a_{1} f f^{\prime}-a_{1}^{\prime} f^{2}-a_{2}^{\prime},
$$

where $a=5 f^{\prime}-\left(F^{\prime} / F\right) f$. From (15), we see that poles of ag come from poles of $f$, poles of $g$ or zeros of $F$. Note that poles of $g$ must be poles of $f$ if they are not poles of $a_{1}, a_{2}$ and $a_{3}$. We only need to consider the poles of $f$ and the zeros of $F$ which are not poles or zeros of $a_{1}, a_{2}$ and $a_{3}$. From (19), we see that any pole of $f$ of multiplicity $k$ is a zero of $a$ of multiplicity $2 k-1$. Such poles of $f$ must be poles of $g$ with multiplicity $k$. Thus poles of $f$ are not poles of $a g$. On the other hand, if a zero of $F$ is a pole of $a$, then it must be a simple pole. From the equation $F=a_{3} g$, we see that zeros of $F$ must be zeros of $g$ if they are not zeros of $a_{3}$. Therefore, zeros of $F$ are not poles of $a g$. Hence $N(r, a g)=S(r, f)$. Note that $m(r, f)=S(r, f)$ and $m(r, g)=S(r, f)$. By Lemma 2, we get $m(r, a)=S(r, f)$. It follows that $T(r, a g)=S(r, f)$. So, $a g$ is a small function of $f$. Let $G=a F$. We have

$$
\begin{aligned}
G & =5 f^{\prime} F-F^{\prime} f \\
& =5 f^{\prime}\left(f^{5}+a_{1} f^{2}+a_{2}\right)-\left(5 f^{4} f^{\prime}+a_{1}^{\prime} f^{2}+2 a_{1} f f^{\prime}+a_{2}^{\prime}\right) f \\
& =\left(3 a_{1} f^{2}+5 a_{2}\right) f^{\prime}-\left(a_{1}^{\prime} f^{3}+a_{2}^{\prime} f\right)\left(3 a_{1} f^{2}+5 a_{2}\right) f^{\prime}-\left(a_{1}^{\prime} f^{3}+a_{2}^{\prime} f\right),
\end{aligned}
$$

and thus $G^{5}=a^{5} F^{5}=a_{3}(a g)^{5} F^{4}=h F^{4}$, where $h=a_{3}(a g)^{5}$ is a small function of $f$. It follows that

$$
h F^{4}=\left(\left(3 a_{1} f^{2}+5 a_{2}\right) f^{\prime}-\left(a_{1}^{\prime} f^{3}+a_{2}^{\prime} f\right)\right)^{5} .
$$


Hence the function

$$
\frac{h F^{4}+\left(a_{1}^{\prime} f^{3}+a_{2}^{\prime} f\right)^{5}}{3 a_{1} f^{2}+5 a_{2}}=: D(f)
$$

is a differential polynomial in $f$. By Lemma 3 we see that $D(f)$ reduces to a polynomial in $f$. Since the numerator of the left-hand side of $(20)$ can be written as $h P\left(f^{2}\right)+f Q\left(f^{2}\right)$, where

$$
\begin{aligned}
& P(z)=z^{10}+6 z^{5}\left(a_{1} z+a_{2}\right)^{2}+\left(a_{1} z+a_{2}\right)^{4}, \\
& Q(z)=4 h z^{7}\left(a_{1} z+a_{2}\right)+4 h z^{2}\left(a_{1} z+a_{2}\right)^{3}+z^{2}\left(a_{1}^{\prime} z+a_{2}^{\prime}\right)^{5},
\end{aligned}
$$

by Lemma 4 we get $P\left(-5 a_{2} / 3 a_{1}\right)=Q\left(-5 a_{2} / 3 a_{1}\right)=0$, i.e.,

$$
\begin{aligned}
& \left(\left(a_{2} / 3\right)^{3}-4(3+2 \sqrt{2})\left(a_{1} / 5\right)^{5}\right)\left(\left(a_{2} / 3\right)^{3}-4(3-2 \sqrt{2})\left(a_{1} / 5\right)^{5}\right)=0, \\
& 6^{3} 5^{5} a_{2}^{3}\left(\left(a_{2} / 3\right)^{3}-4\left(a_{1} / 5\right)^{5}\right) h+3\left(3 a_{1} a_{2}^{\prime}-5 a_{1}^{\prime} a_{2}\right)^{5}=0 . \\
& \text { (21), }
\end{aligned}
$$

From (21), we see that $a_{2}^{3} / a_{1}^{5}$ is a constant, and thus $3 a_{1} a_{2}^{\prime}-5 a_{1}^{\prime} a_{2}=0$. Then from (22) we get $h=0$, a contradiction. Hence equation (15) has no nonconstant meromorphic solution. This completes the proof of Theorem 1.

\section{References}

[1] J. Clunie, On integral and meromorphic functions, J. London Math. Soc. 37 (1962), $17-27$.

[2] W. Doeringer, Exceptional value of differential polynomials, Pacific J. Math. 98 (1982), 55-62.

[3] I. Laine, Nevanlinna Theory and Complex Differential Equations, de Gruyter, New York, 1993.

[4] P. Li and C.-C. Yang, Admissible solution of a functional equation of Diophantine type, in: Some Topics on Value Distribution and Differentiability in Complex and p-adic Analysis, Science Press, Beijing, 2008, 250-264.

[5] - - - Meromorphic solutions of functional equations with nonconstant coefficients, Proc. Japan Acad. Ser. A 82 (2006), no. 10, 183-186.

[6] C.-C. Yang and P. Li, Some further results on the functional equation $P(f)=Q(g)$, in: Value Distribution Theory and Related Topics, Adv. Complex Anal. Appl. 3, Kluwer, Dordrecht, 2004, 219-231.

[7] H.-X. Yi, Some results concerning differential polynomials, Acta Math. Sinica 33 (1990), 302-308 (in Chinese).

Department of Mathematics

University of Science and Technology of China

Hefei, Anhui, 230026, P.R. China

E-mail: pli@ustc.edu.cn

Received 2.5.2008

and in final form 26.6.2008 